\title{
Advances in medium access control standards for cognitive radio
}

\author{
Danilo A. López S $\mathrm{S}^{\# 1}$, Camilo A. Rojas ${ }^{\# 2}$, Diego F. Zapata ${ }^{\# 3}$ \\ ${ }^{\#}$ FullTime Professor at Universidad Distrital Francisco José de Caldas, \\ Faculty of Engineering, Bogotá (Colombia-South America) \\ dalopezs@udistrital.edu.co

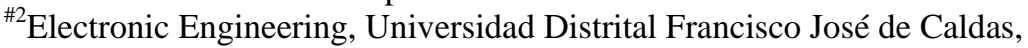 \\ Faculty of Engineering, Bogotá (Colombia-South America) \\ canzolar@udistrital.edu.co \\ \#3 Electronic Engineering, Universidad Distrital Francisco José de Caldas, \\ Faculty of Engineering, Bogotá (Colombia-South America) \\ fernandoz6m4@gmail.com.co
}

\begin{abstract}
The article intends to make a succinct compilation of the advances made in the control of medium access in cognitive radio systems, in addition to presenting a classification system of secondary cognitive users based on the classification of SUs with FAHP, in order for the allocation of resources in the stage of spectral sharing of cognitive radio networks to be fairer or more equitable. To estimate the level of performance for the "equitability" metric, several simulations were performed and the behavior of the following metrics was evaluated: admission efficiency, efficiency of amount of information sent, energy efficiency and total efficiency.
\end{abstract}

Keyword- MAC-CR, PUs, SUs, Cognitive radio, FAHP, algorithm.

\section{INTRODUCTION}

One of the most effective modern techniques to mitigate the impact of the spectrum shortage problem is cognitive radio (CR) [1], whose foundation is to take advantage of the underused bands in terms of time and space to allow unlicensed or secondary users (SU) to access the licensed spectrum when and where these bands are not occupied.

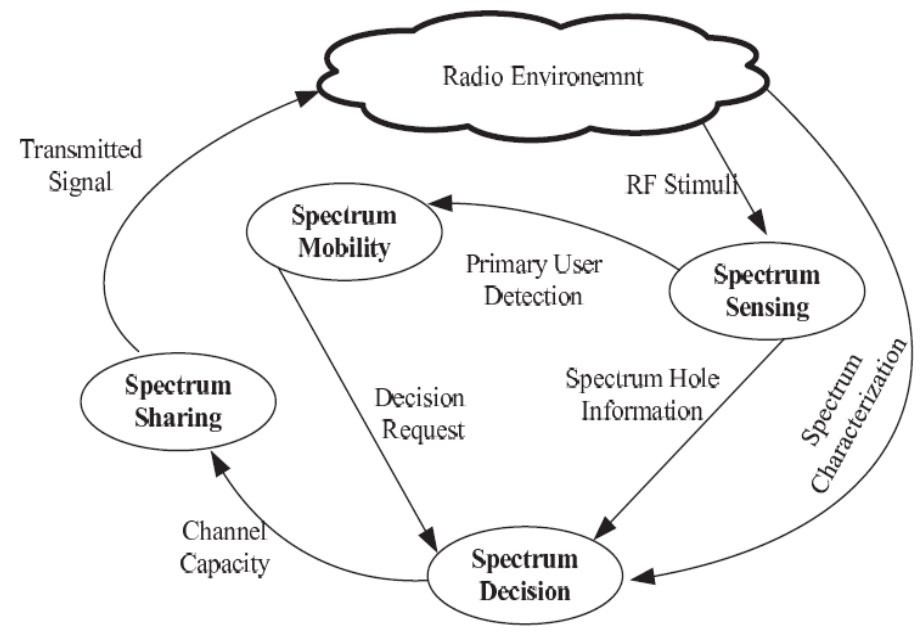

Figure 1. Dynamic spectrum management framework in CR [2].

Taking Figure 1 as reference, one of the fundamental stages of CR relates to "Spectrum Sharing", which refers to the ability of cognitive radios to efficiently and equitably allocate the resources reserved in the "Spectrum decision" stage; In this sense, the article aims to present the advances in the development of proposals aimed at efficient resource allocation; In addition to complementing the proposal presented in [3] for the implementation of the MAC-CR algorithm, which seeks to allow secondary users the most equitable access possible without neglecting the search for maximum free-band use. For the classification of SUs at channel allocation, the design of the FAHP system (Fuzzy Analytic Hierarchy Process) shown in [3] was studied in depth. In order to observe the system's functioning, a simulation of the algorithms was performed in Matlab assuming that the other phases of the CR scheme (spectrum sensing, PU characterization [4], spectral decision [5] and spectral mobility) work perfectly so that errors in these stages would not affect the evaluation of the model. 


\section{ADVANCES IN MAC STANDARDS FOR COGNITIVE RADIO}

In [6] the authors present the CRUAM-MAC model, which allows to dynamically assign the transmission channels of the spectrum,thus solving the distribution of resources to the CR terminals using an overlay approach in order not to interfere with PU transmissions. However, it uses the underlying method to perform the process of establishing the communication channel between a CR pair. The use of the latter technique allows disposing of a CCC, but causes minimum interference in the channel during the connection process. The duration of the interference depends on the time it takes to perform the spectrum monitoring process and the exchange of messages between a pair of terminals to choose a channel that is available and common to both. Once a channel is chosen, CRs consider other free channels as possible backup transmission media. If a SU is interrupted by a PU, the protocol's mobility mechanism allows the terminal to move the transmission to another channel from the list of available channels to continue the communication. Due to this, it is of great importance that the list of available channels be updated periodically according to status changes in the available spectrum. In case a channel change has to be made, the devices use a 4-byte coordination message to agree on a new channel in order to continue communication. The sending of this coordination message is done in the underlying mode (underlay) causing minimum interference that is equivalent in time to the 4 bytes of its size divided by the value of the channel transfer rate [3].

In the proposal for spectrum sharing described in [7], the primary link has a high priority for transmitting data in relation to the secondary link. As long as the quality of service (QoS) of the PU is maintained, the SU will be able to use the spectrum. SU power is regulated to maintain the QoS of the primary link. For the intelligent assignment of SU power, a technique based on ANFIS (Adaptive Neuro Fuzzy Inference System) was used. To make optimal considerations regarding power management and allocation to SUs, metrics such as the signal to noise ratio (SNR) for non-interference media, and the signal to interference and noise ratio (SINR) for media with interference, (in this case caused by the SUs), were taken into account. Another relevant factor was the relative distance between SUs and PUs; When the relative distance is small, the interference caused by the cognitive link will be high and so the SU must transmit at low power. When the relative distance is large, then the interference caused by the cognitive link will be low, so the SU can transmit with the maximum power scale [3].

According to what was proposed by [8], most of the research that works on spectrum sharing does not take into account the heterogeneity of the spectrum, as well as different transmission ranges, error rates of each channel, etc. In [9] an algorithm is presented which adapts to the heterogeneous spectrum for a centralized network topology, taking SUs as mobile, where each SU is assigned a channel considering its occupation time, its position and an opportunity factor of access to the right channel, which is a relationship between the number of times it was granted access to a channel and the number of times it was blocked access. The total capacity of the network depends mainly on the spectrum sharing scheme [10]; in this sense there are two models of spectrum sharing: exclusive allocation and common use; the first one assigns a channel to a single SU when it requires it, which leads to a problem of unequitability in the allocation of channels, while in the 'common use' schemeseveral SUs are allowed to use a channel at the same time,thus controlling the power level of each SU [11]; Network capacity is lower with this latter scheme due to co-channel interference, but mitigates allocation unequitability. In previous works only one of the two sharing schemes is taken into account, [12] works with both schemes in inter-network and intra-network topologies [3].

The thesis developed by [13] proposed the deployment of a protocol that allows to coordinate channel access in an efficient way avoiding the interference between users; for this, a simulation software was implemented that allows to establish the advantages of using the protocol, among others, a mobility mechanism in the presence of the PU, rapid package transfer and greater medium access speed. For the development of the protocol, a combination is made between the characteristics of distributed cognitive networks and the standard for wireless LANs. Among the main results is the complete solution for cognitive radio devices to access the spectrum, without requiring the use of a common control thanks to monitoring of the network, without generating alternate power consumption and with coordinated communication between the same cognitive radio devices. Regarding the behavior of simulations of the different stages, it meets the project's objectives, showing that thanks to the protocol and counting or not with the provision of backup channels, access to the medium and the coordination of channel changes due to the presence of PUs, considerably improvesand speeds up the transfer of data packages with the help of interval waiting between frames, which gives the work greater wireless medium access and data transfer speed.

In the research carried out in [14], the authors address the design of an efficient spectrum allocation mechanism in cognitive radio networks, developing a framework that allows for the auction of SUs to share the spectrum available, when the PU is absent. Each PU is the resource provider for the announcement of a price and a reserve offer and each SU acts as a client; As there are several SUs, they will be forced to compete in a noncooperative auction game (formulated by the authors). The authors also focus their study on establishing PU prices to maximize their income; so they propose a learning algorithm to set these prices, taking into account 
that the income of each PU must be proportional to the interference. Consequently, the results show that the learning algorithm can converge in a balanced way with reasonable efficiency in the distributed network; they also conclude that the proposed auction framework has a high degree of efficiency and balance in terms of spectrum allocation.

In [15], the authors are responsible for implementing the multiuser load balancing in CR, thus distributing the traffic loads of secondary users to multiple channels, therefore proposing an analytical model that evaluates the multiple interruptions that occur when the PU connects, loss of detection and false alarm for SUs; maximizing the probability for the selection of the optimal channel for SUs. Based on this analytical model, the optimal number of candidate channels for the selection method based on spectrum detection and the probability of choosing the optimal channel for the probability-based selection method can be obtained analytically for various detection time and traffic parameters. It was found that the probability-based regime can reduce the total system time compared to the detection-based regime, when the secondary user's traffic loads are light, while the detection-based regime offers better performance in the condition of heavy traffic loads. This observation provides an important advance in the design of an adaptive traffic spectrum decision and sharing scheme in the presence of detection errors.

The article presented in [16]titled ANFIS based opportunistic power control for cognitive radio in spectrum sharing aims to strike a balance between minimizing PU interference and improving SU performance; To attack each problem, the authors consider the variation of SU transmission power and the maintenance of PU quality of service (QoS), respectively. To that end, they implemented an opportunistic control strategy based on an Adaptive Neuro-Fuzzy Inference System (ANFIS) as a power control strategy and the signal-to-noise ratio (SNR) of the PU that is defined as the noise power that interfereswith the power of the signal being transmitted and the gain of the PU interference channel as inputs. In this way the results confirm that the error rate of the proposed strategy is lower than the rate without power control; it is for this reason that the authors affirm that ANFIS can be used efficiently for power control in CR since it improves SU performance, reducing efforts, learning and adapting from the conditions given by the environment.

The article Intelligent Wireless Communication System Using Cognitive Radio in [17], deals with the problem of spectrum allocation and the guarantee of a better management seeking to improve wireless communication for a single mobile radio cognitive terminal; for this, they propose the use of a multi-agent system (MAS) to access the spectrum cooperatively (agents with common interests) and in competition. The authors propose an agent-based architecture, where each CR terminal must contain an intelligent agent. The information collected (from the radio environment) will be stored in a shared knowledge base that all agents can access, in order to increase their collective and individual gain; there the SU agent shall send messages according to the PU agent and this in turn must respond accordingly, so that they can share the spectrum; In case of interferences, the authors propose protective measures by controlling the transmission power of the cognitive terminal; in this way, the radio can foresee the difference in coverage and thus, know the necessary signal from the base station (BS), to reconfigure its characteristics. In this case the database will be divided into two parts, the first for learning and the second for testing, this will be measured in terms of performance of a mobile user videoconference. According to the simulations carried out, it is established that regardless of the number of correspondence agreements established between the SU and the PU agent, using the MAS will make spectral sharing in the CR environment efficient.

In [18] radio and spectrum management resources are analyzed to provide maximum and efficient use of spectrum resources with the overall objective of maximizing user capacity while ensuring QoS (Quality of Service) restrictions, focusing mainly on how to select the appropriate Radio Access Technology (RAT) after the start of the session in a heterogeneous access network. They concluded that in scenarios with limited access due to coverage, availability of terminals and RAT service compliance, two key parameters were identified that influence the performance of the RAT selection. First, the relationship between the demanding services greatly affects the suitability of a selection process based on a particular service. Second, the processing of resources between services in a single RAT, compared to RATS that only support one type of service, causes greedy service allocation principles.

\section{SU CLASSIFICATION MODEL IN THE SPECTRUM SHARING STAGE IN CRNS.}

Taking as reference the article in [3] in which the MAC standard model called CRUD-MAC was implemented, it then shows the way in which the FAHP methodology was used for SU classification when assigning the channels selected in the spectrum decision phase.

A.FAHP algorithm hierarchical structure.

From the objective, criteria and alternatives, which are discussed in [19], the hierarchy was built, with which the rest of the proposed FAHP algorithm design methodology was developed, which can be seen in Figure 2. 


\section{B.Judgment Matrix for each criterion.}

Once the hierarchy was constructed (shown in Figure 2), the judgment matrix was generated, which corresponds to comparative evaluations that define the level of relative importance between each possible combination of pairs of criteria. Because this comparison requires a broad and clear knowledge of the subject, a survey was designed with the objective that experts in the field made their respective comparative judgments. Each of the experts was surveyed independently, ensuring that the responses of others did not influence their judgment. To determine the final judgment matrices, the average preference values issued by the individuals was used.

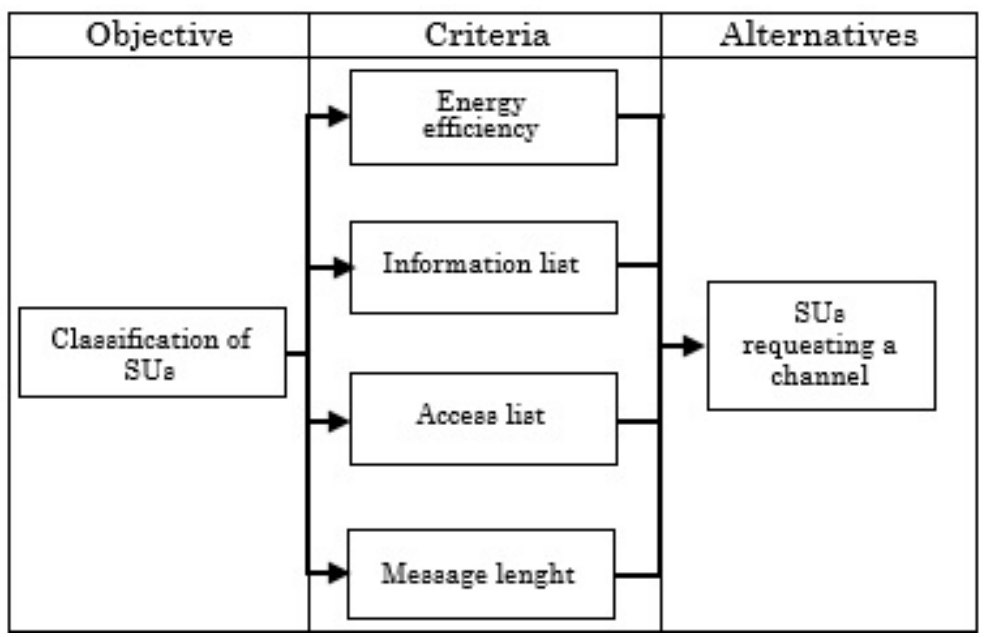

Figure 2. Dynamic spectrum management framework in CR [3].

Because the fuzzy numbers do not define a specific number but a range of values, to calculate the normalized weights and the degree of consistency of the judgment matrix, the median or average of the fuzzy triangular number was used. With the use of the average number, the validation of the linearity and consistency of the judgments is done to validate the AHP method, without affecting the final result of the decision analysis by the FAHP method.

For example, for the calculation of the relative importance value between the Information ratio and Energy efficiency criteria, the importance intensity of the surveys carried out was obtained, namely DMAI, DMAI and FMEI. To have a specific number, the fuzzy triangular average number was used, being 1.5 for DMAI and 0.5 for FMEI according to [20], and with specific numbers for relative importance, the average of the sum of the values 1.5, 1.5 and 0.5 was used, throwing a result of 1.16 and this is the value shown in the matrix of Table 1 for the relationship between the "Information ratio" and "Energy efficiency" criteria, and in the same way for the rest of the values in the matrix. The judgment matrix for the criteria specified above is shown in the same table. The diagonal of the matrix is the unit because the importance of each criterion is being compared with itself. The upper diagonal half of the matrix describes the relative importance of the criterion of the first column with respect to the criterion of the first row.

TABLE I. Judgment Matrix for selected criteria.

\begin{tabular}{|c|c|c|c|c|}
\hline Criteria & $\begin{array}{c}\text { Energy } \\
\text { Efficiency }\end{array}$ & $\begin{array}{c}\text { Information } \\
\text { Ratio }\end{array}$ & $\begin{array}{c}\text { Access } \\
\text { Ratio }\end{array}$ & $\begin{array}{c}\text { Message } \\
\text { Length }\end{array}$ \\
\hline Energy Efficiency & 1 & 0.862 & 0.642 & 1.466 \\
\hline Information Ratio & 1.16 & 1 & 0.666 & 1.333 \\
\hline Access Ratio & 1.5555 & 1.5 & 1 & 1.722 \\
\hline Message Length & 0.681 & 0.75 & 0.580 & 1 \\
\hline
\end{tabular}

\section{C.Normalized weight for each criterion.}

The calculations of the normalized weights for the criteria were obtained according to that shown in [21] and the results are shown in Table 2. 
TABLE II. Normalized weight for all criteria.

\begin{tabular}{|c|c|}
\hline Criteria & Normalized Weight \\
\hline Energy Efficiency & 0.23 \\
\hline Information Ratio & 0.25 \\
\hline Access Ratio & 0.34 \\
\hline Message Length & 0.18 \\
\hline
\end{tabular}

The normalized weights describe the degree of relative importance of each criterion for the classification of SUs; and in accordance with what is found in Table 2, said classification will depend by $34 \%$ on the access ratio, $25 \%$ on the information ratio, $23 \%$ on energy efficiency and $18 \%$ on message length.

With the weights described in Table 2, all the SUs that sent a transmission request to the BS were evaluated, which are part of the alternatives in the hierarchy of the developed FAHP algorithm and were then ranked from highest to lowest score.

After classifying SUs in a single (global) list and with the list of classified channels obtained from the spectral decision stage, the channel and power allocation algorithm comes into operation, starting with the first SU in a list of classified secondary users and in descending sequence, this happens until all the channels that were initially available and still available are assigned; when this happens it is counted as a failed attempt for the SUs that are still in the queue waiting for assignment and no channel was assigned to them after all the channels are assigned or channels are assigned to all the cognitive nodes, the assignment round ends, and the BS sends a new broadcast to perform a new classification and assignment.

D.Judgment matrix consistency index.

To evaluate the validity of the developed FAHP algorithm, the consistency index was calculated as shown in Table 3. As its value is less than 0.10 , the development of the algorithm is satisfactory and it can be used.

TABLE III. Judgment matrix consistency index.

\begin{tabular}{|c|c|}
\hline $\begin{array}{c}\text { Judgment } \\
\text { Matrix }\end{array}$ & $\begin{array}{c}\text { Consistency } \\
\text { Matrix }\end{array}$ \\
\hline Best Effort & 0.062 \\
\hline
\end{tabular}

\section{IV.RESULTS OBTAINED FROM SU CLASSIFICATION.}

The system evaluation within the CRUD-MAC system presented in [3] was carried out for the cases in which a classification of SUs was not included (Section 4.1) and those in which a classification with FAHP was included (Section 4.2) under the premise that the number of channels available in each broadcast generated (or broadcast) was 100 and the number of SUs that arrived at the central station requesting a channel increased rapidly (it starts at 21 SUs in the first access and reaches up to 500 SUs in the sixth broadcast generated). Specifically, the characteristics of the simulation carried out in Matlab were:

Total number of channels = 200;

Number of free channels per broadcast $=100$;

Total number of SUs = 500;

Number ofbroadcasts $=100$;

\section{A.Unclassified results.}

In order simulate the system without classification, a list of SUs with a variable random message length was generated for each SU when the broadcast changed (the efficiencies are obtained from historical results and initially they are zero) and they were assigned channels in random order, unlike the classifier systems that allocate resources to SUs in an established order. To evaluate the performance of the system taking into account that an equitable or fair allocation of resources is sought, a performance metric was used for each SU and it was sought that the value of this metric was the highest and at the same time as uniform as possible for all SUs in such a way that a good performance is guaranteed in the use of resources as well as the fairness among users; In order to achieve this, the following evaluation variables were defined:

1) Efficiency in admissions of each SU: Refers to the relationship that exists between the number of times the SU in particular has achieved satisfactory access to the spectrum and the number of total attempts to do so.

2) Efficiency in the amount of information sent from each $S U$ : Refers to the relationship between the amount of information that the SU in particular has successfully transmitted and the total amount of information that it has attempted to transmit. 
3) Energy efficiency of each SU: Establishes the relationship between the energy that the SU would have consumed if it had been assigned the best channel in all assignments, and the total energy consumed by the SU in its access history.

4) Total efficiency of each SU:Defined as the average of the three efficiencies mentioned above. The mathematical definitions of these evaluation metrics are found in [3].

As can be seen in Figures 3 to 6, the historical efficiencies of the SUs are relatively low, due to the fact that the number of SUs grew rapidly, causing the available channels to not be able to handle all the requests.

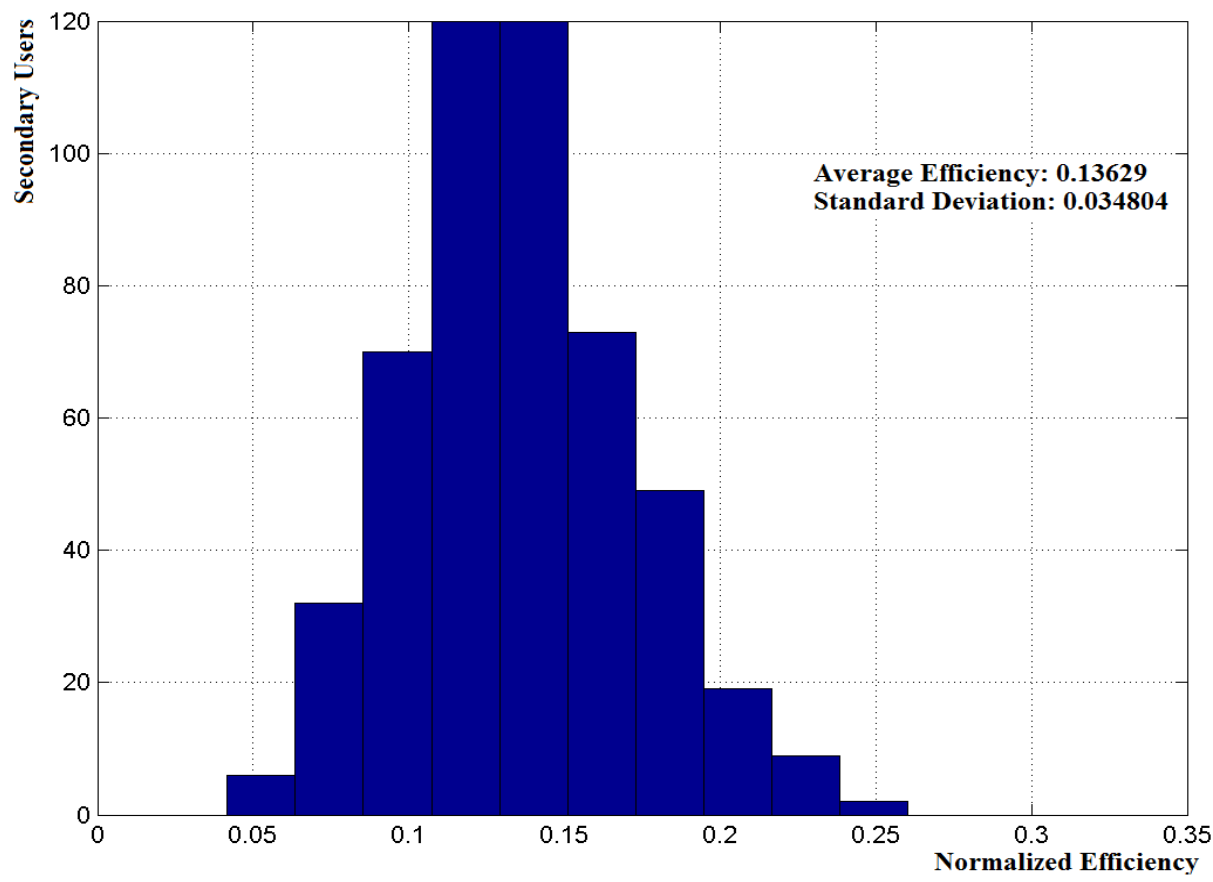

Figure 3. Unclassified admission efficiency histogram.

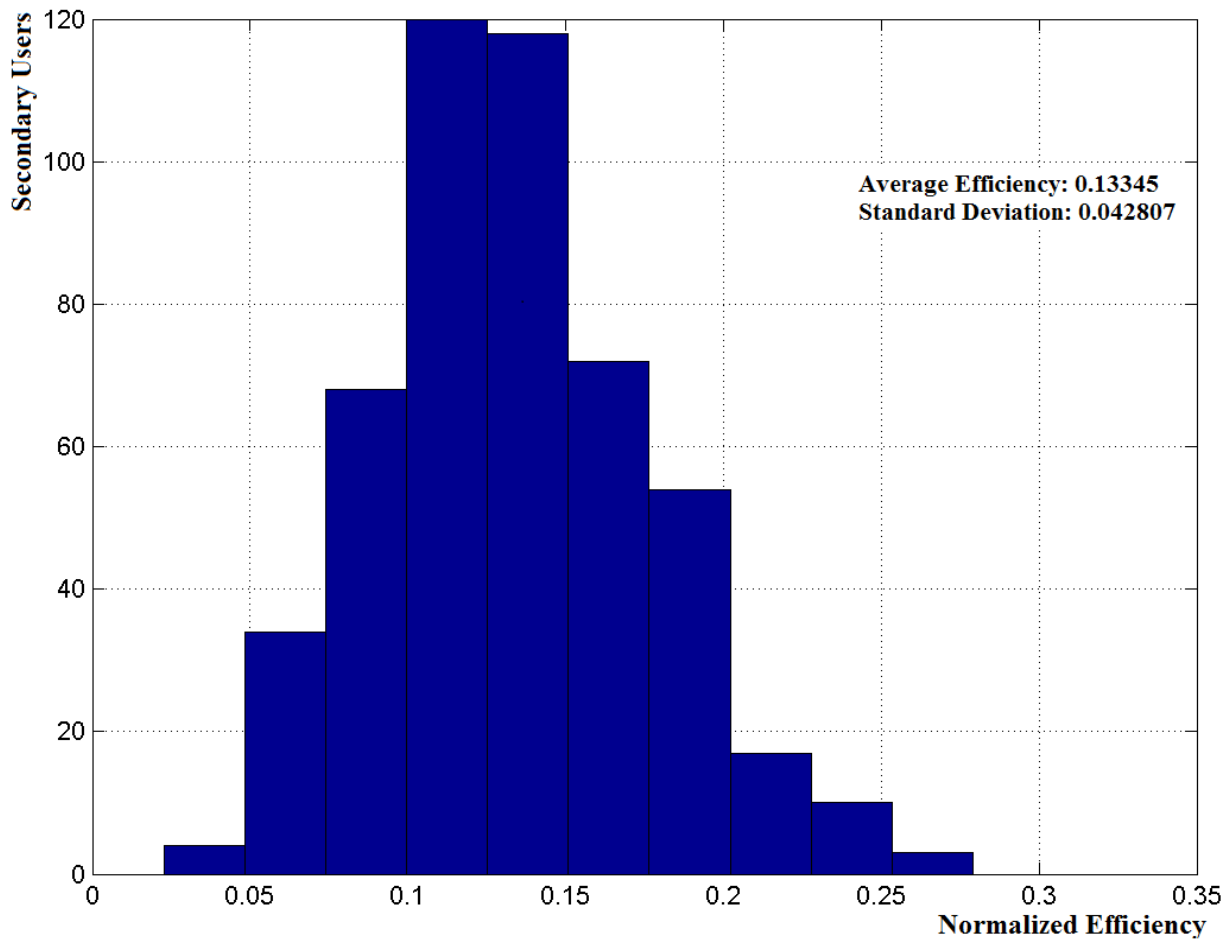

Figure 4. Unclassified information ratio efficiency histogram. 


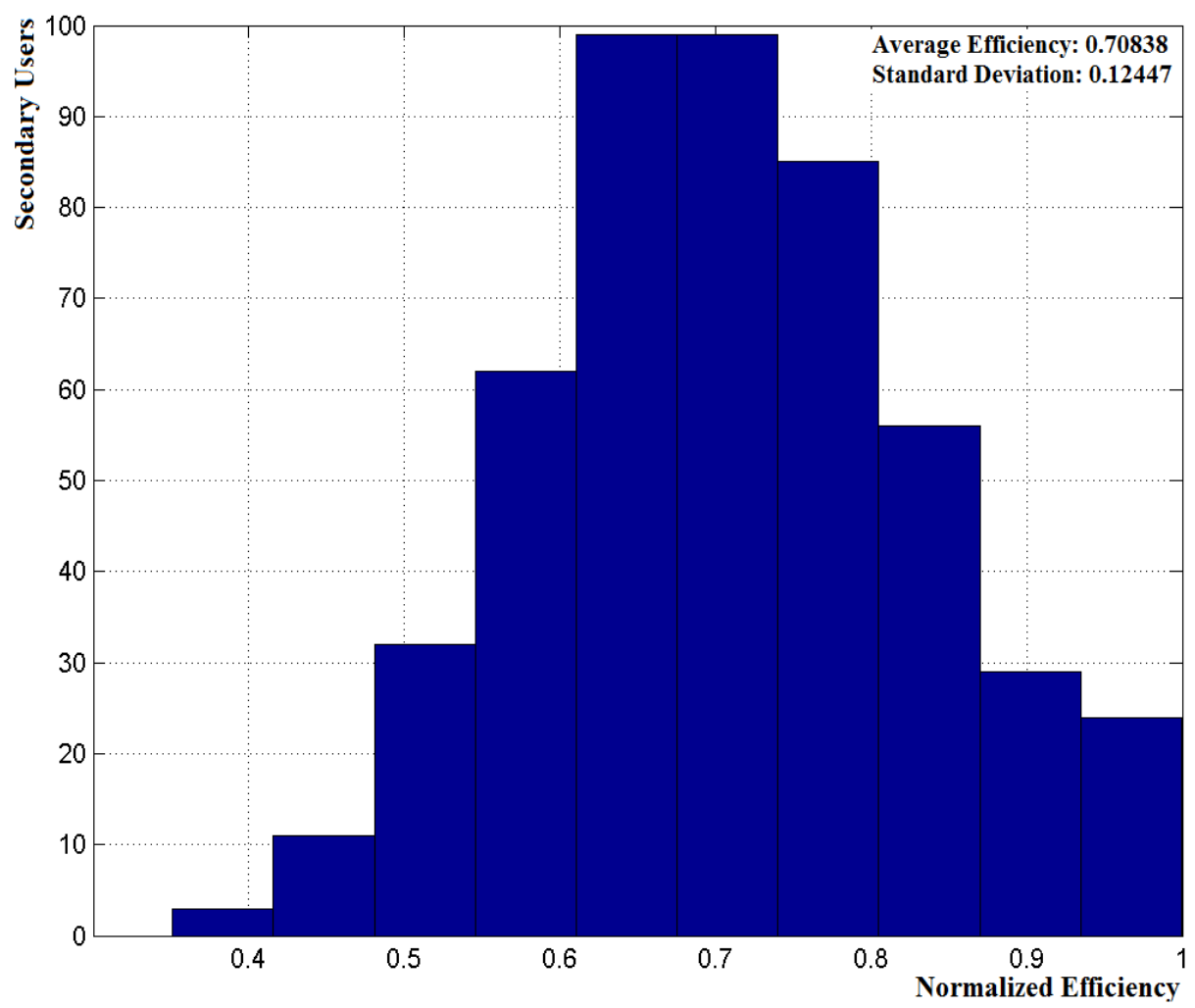

Figure 5. Unclassified energy efficiency histogram.

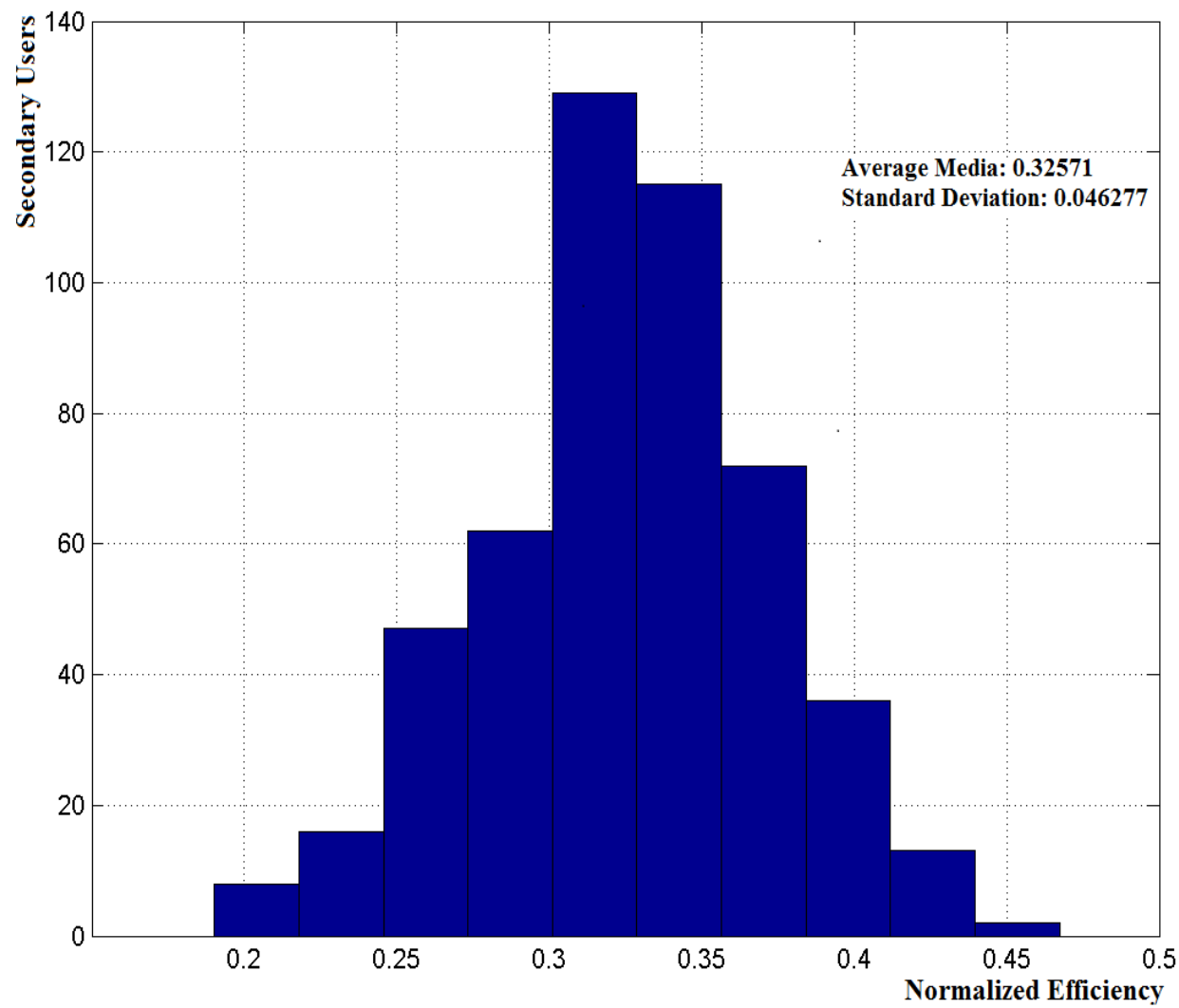

Figure 6. Total efficiency histogram.

In Figure 7, the behavior of the amount of SUs accepted and rejected is shown, as in this test the number of SUs grows rapidly it can be observed that the amount of SUs accepted departs early from the trend of total SUs and likewise the number of rejected SUs grows suddenly. 


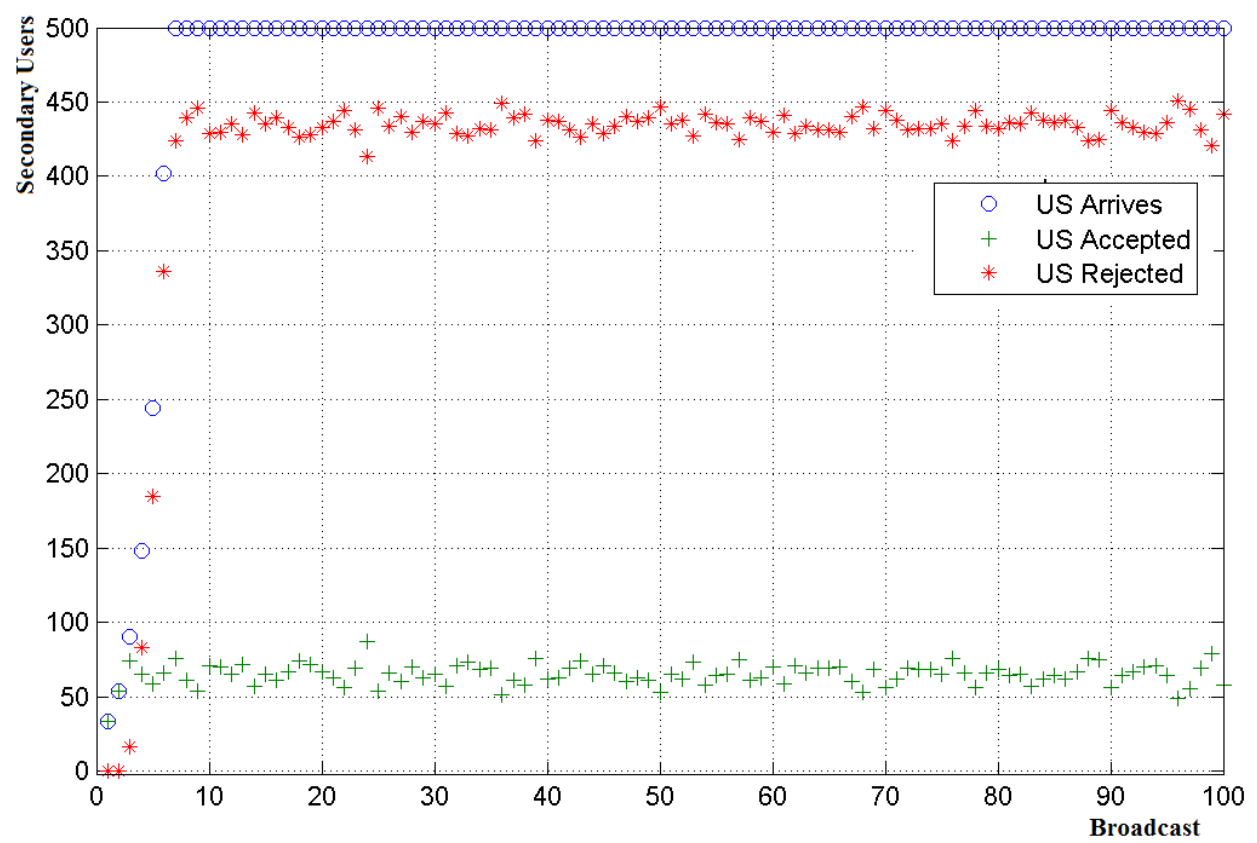

Figure7.Accepted and rejected SUs for the unclassified case.

B.SU classification results using FAHP.

The results obtained by applying the SU classifier are shown in Figures 8 through 11.

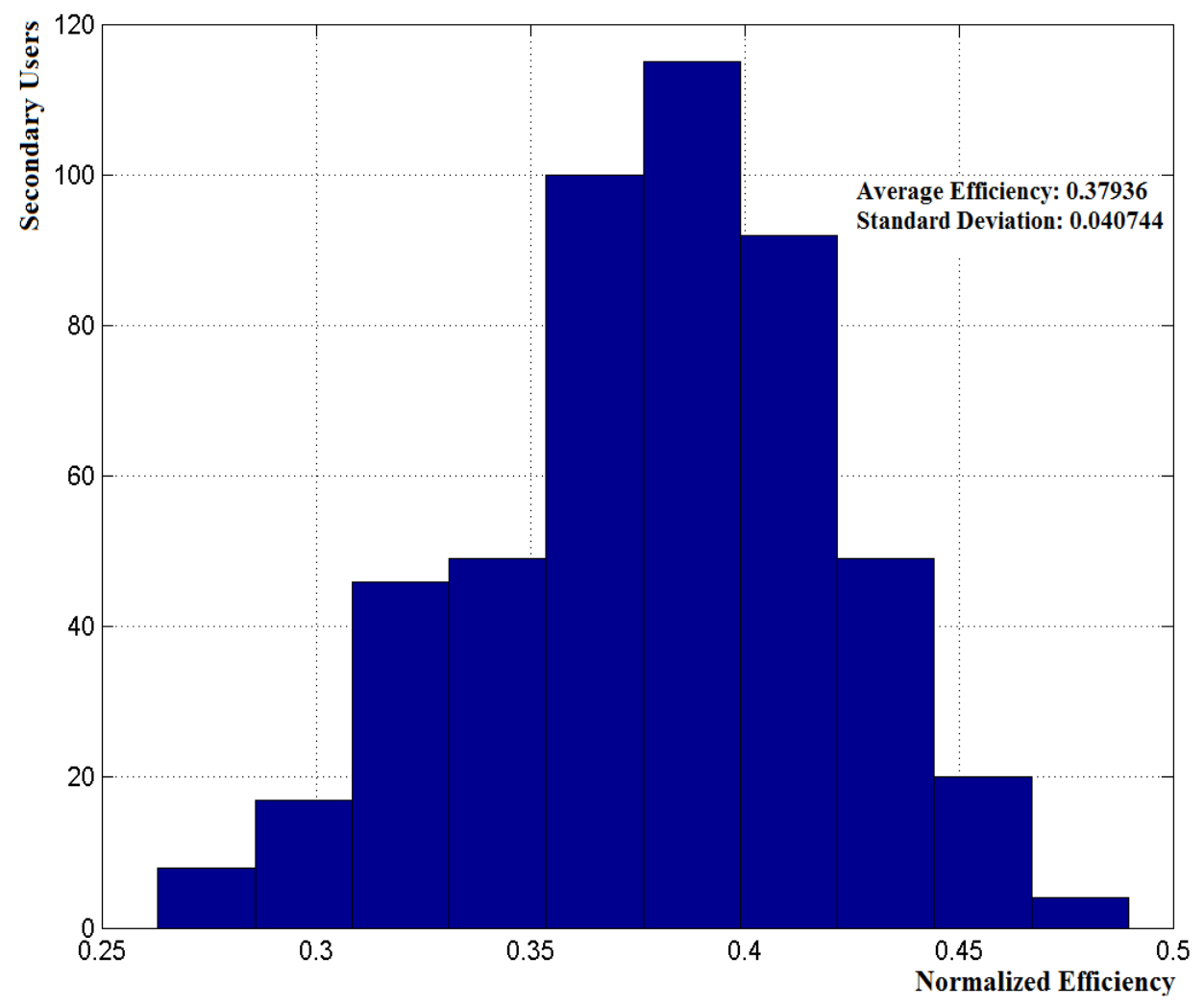

Figure 8. Admissions efficiency histogram with FAHP classifier. 


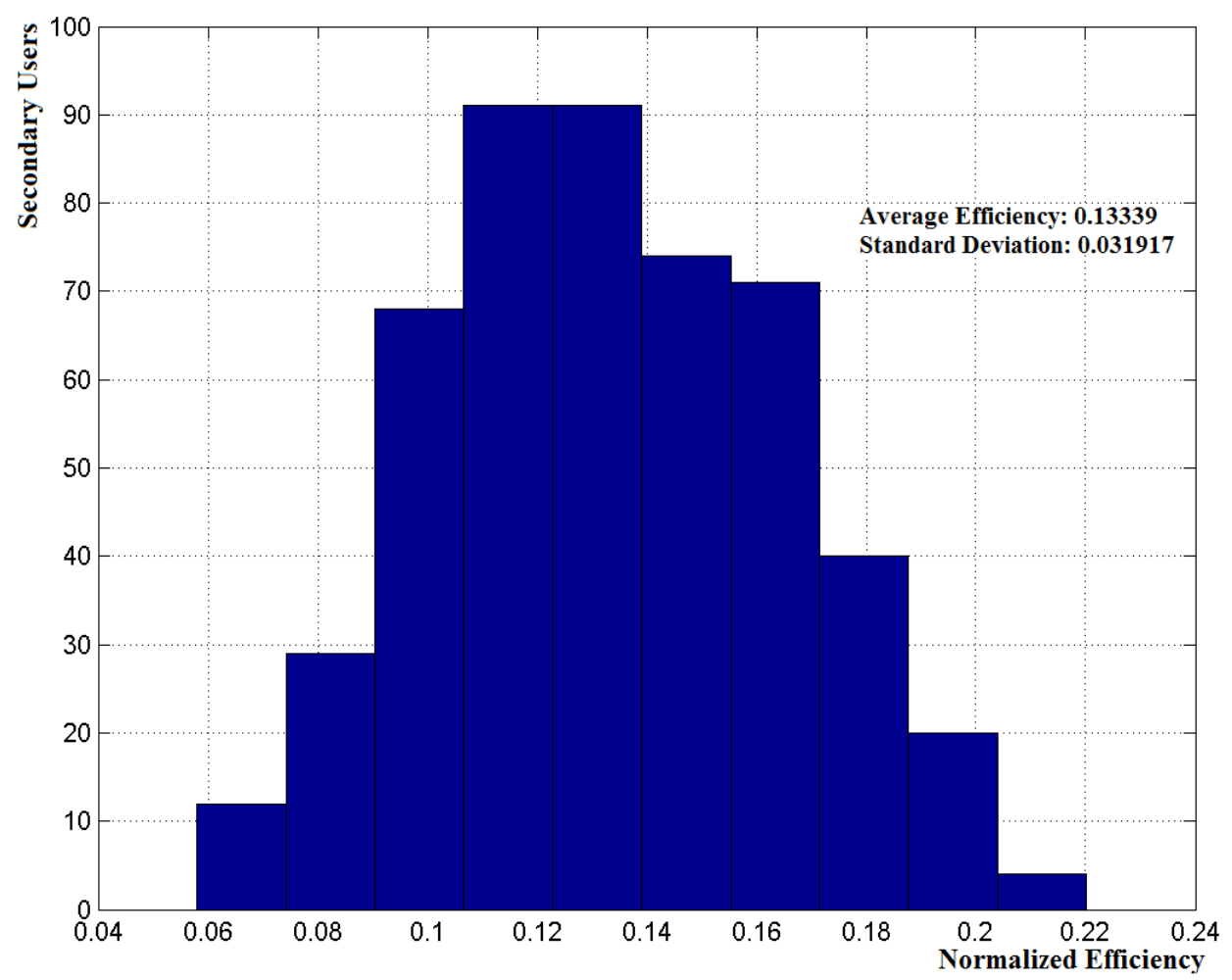

Figure 9. Information ratio efficiency histogram sent with FAHP.

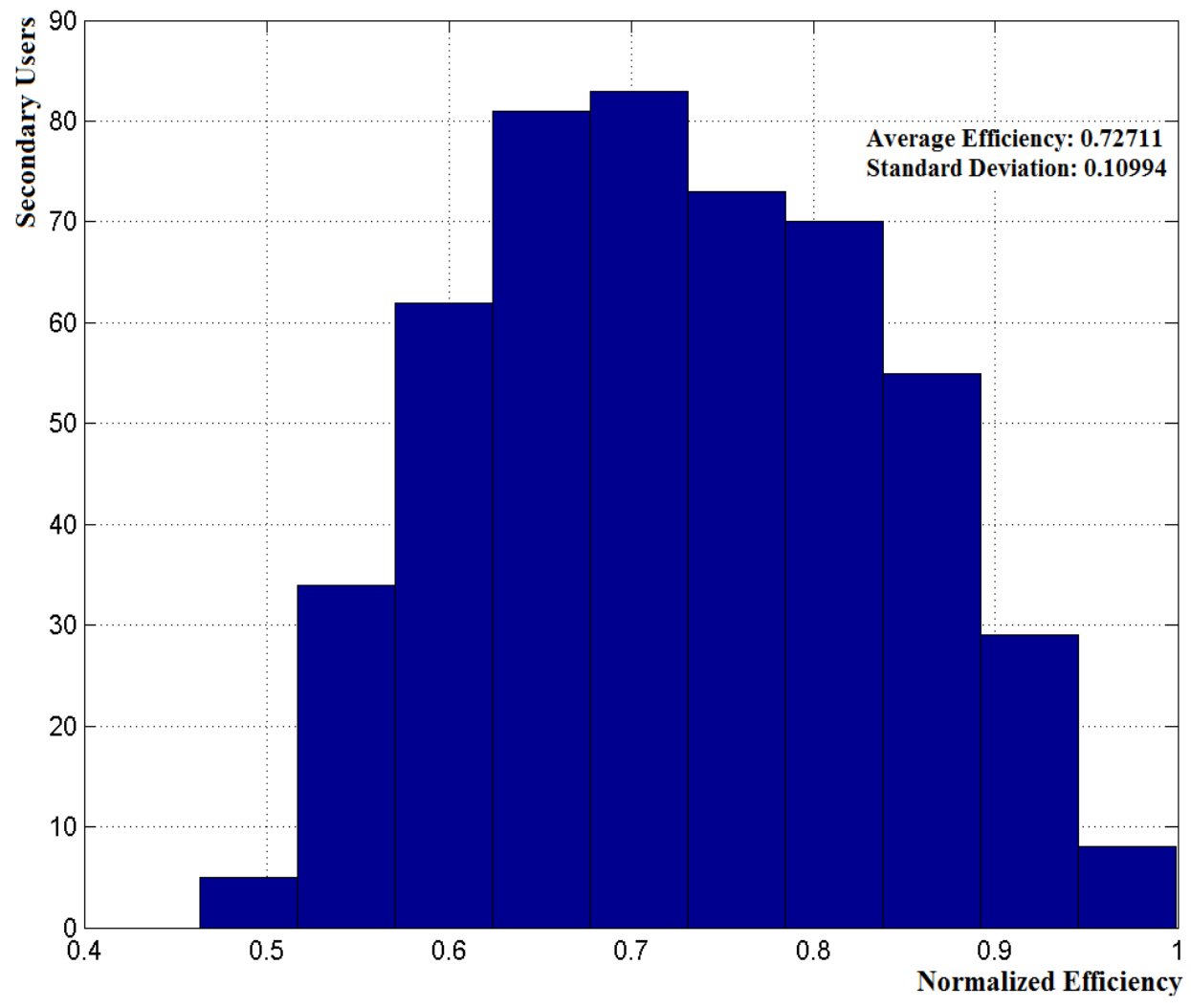

Figure10. Unclassified energy efficiency histogram. 


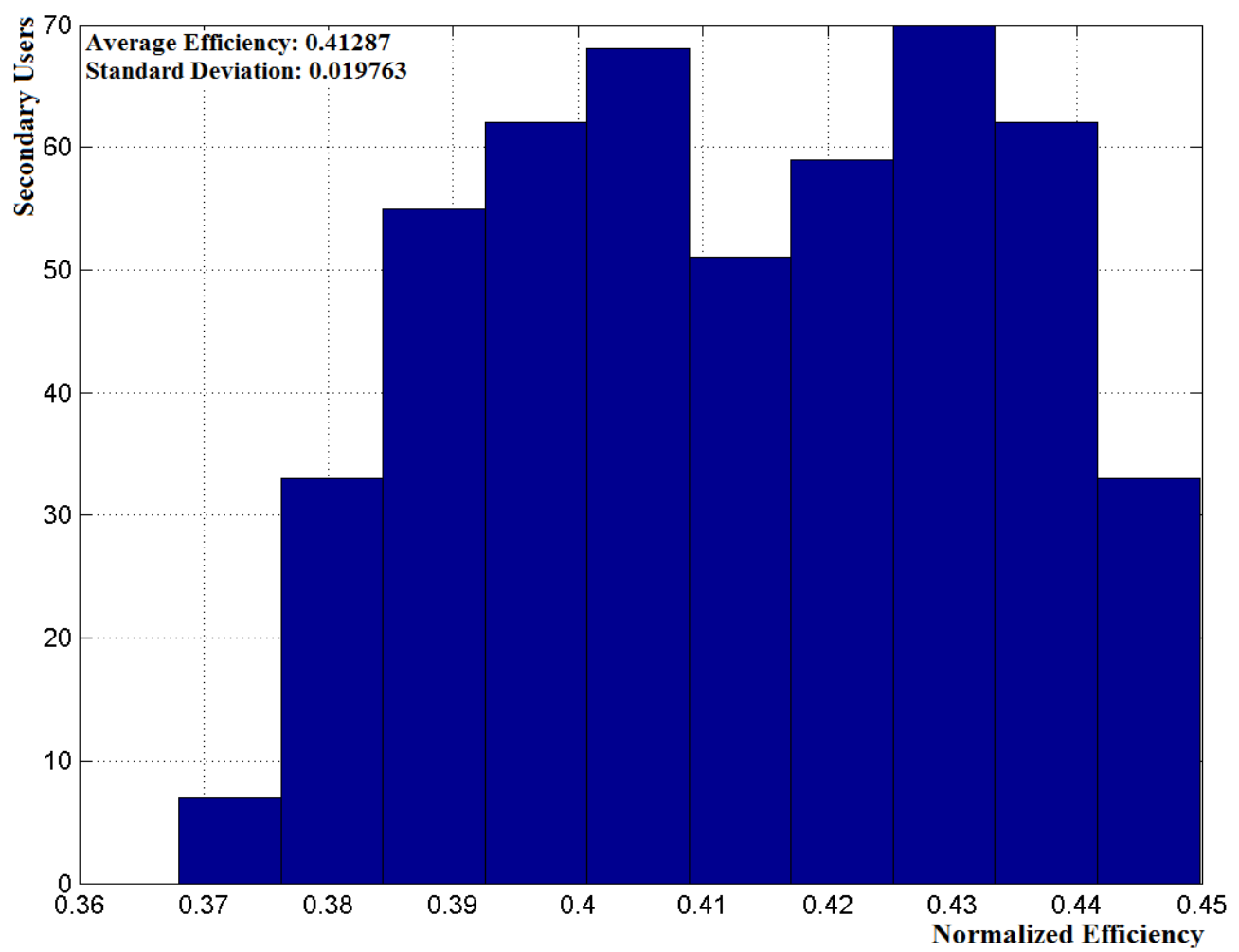

Figure11. Total efficiency histogram.

It can be seen that when comparing the histograms individually, in most cases an improvement was obtained in both the average efficiency (increased) and the standard deviation (decreased) with respect to the unclassified test (Section 4.1).

Figure 12 shows the behavior of the amount of SUs accepted and rejected, as in this test the number of SUs grows rapidly it can be seen that the amount of SUs accepted departs early from the trend of total SUs and likewise the amount of rejected SUs grows suddenly.

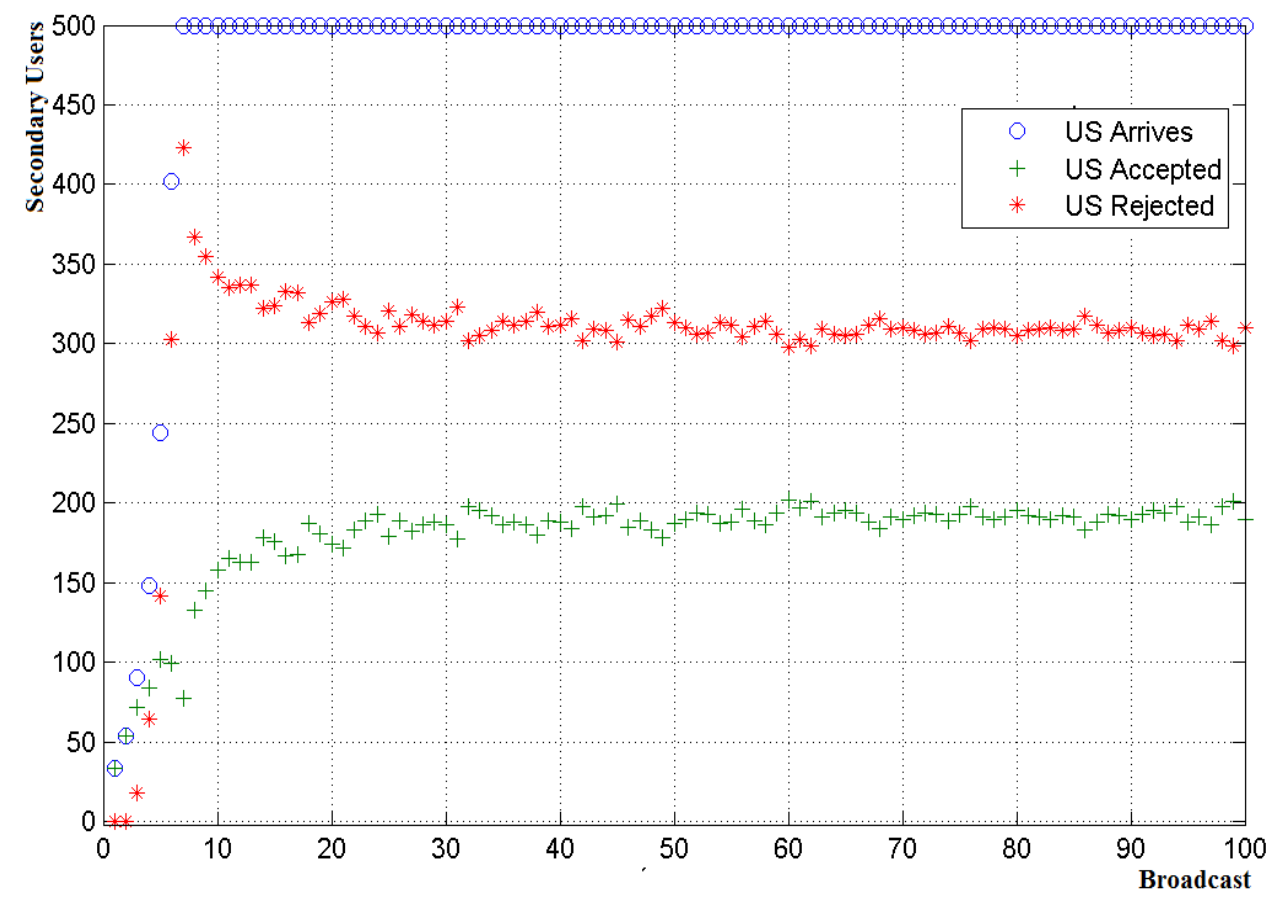

Figure 12.Accepted and rejected SUs with FAHP classification.

Table 4 shows the summary of the results obtained for the second test with respect to the mean and the standard deviation of the total efficiency, it is worth remembering that what was sought is to minimize the "standard deviation" and maximize the "average", an objective that was achieved. 
TABLE IV. Comparison of average efficiency and standard deviation for the test cases.

\begin{tabular}{|c|c|c|c|c|c|c|c|c|}
\cline { 2 - 9 } \multicolumn{1}{c|}{} & \multicolumn{2}{c|}{$\begin{array}{c}\text { Admission } \\
\text { Efficiency }\end{array}$} & \multicolumn{2}{c|}{ Information Ratio } & \multicolumn{2}{c|}{ Energy Efficiency } & \multicolumn{2}{c|}{$\begin{array}{c}\text { Average } \\
\text { Efficiencies }\end{array}$} \\
\hline Test & Mean & $\begin{array}{c}\text { Standard } \\
\text { Deviation }\end{array}$ & Mean & $\begin{array}{c}\text { Standard } \\
\text { Deviation }\end{array}$ & Mean & $\begin{array}{c}\text { Standard } \\
\text { Deviation }\end{array}$ & Mean & $\begin{array}{c}\text { Standard } \\
\text { Deviation }\end{array}$ \\
\hline Unclassified & 0.136 & 0,034 & 0.133 & 0.042 & 0.708 & 0.124 & 0.325 & 0.046 \\
\hline FAHP & 0.379 & 0,04 & 0.133 & 0.031 & 0.727 & 0.109 & 0.412 & 0.019 \\
\hline
\end{tabular}

\section{Conclusions}

From the tests carried out it was possible to demonstrate that the implementation of a classifier system within the spectrum sharing process in cognitive radio, can have advantages since it is possible to obtain an increase in the number of SUs that can access the spectrum and a decrease of unattended cognitive nodes, thus achieving greater equitability in the existing resources in cognitive networks. In addition improvements were obtained regarding quantitative measures defined as evaluation metrics: an increase in the mean and a decrease in the standard deviation.

In the event that the available channels are sufficient for all the SUs to carry out their communications without problem, the implementation of a classification system is not considered essential, because even without it all SUs will have access to the spectrum and, on the contrary, if it is implemented it can be considered an unnecessary effort.

\section{REFERENCES}

[1] J. Mitola, G. Maguire, Cognitive radio: making software radios more personal, IEEE Personal Communications, Volume 6, Issue 4, pp. 13-18, 1991.

[2] M. Masonta, M. Mzyece, N. Ntlatlapa, Spectrum decision in cognitive radio networks: A survey, IEEE Communications Society, Communications Surveys \& Tutorials 2013, Volume 15, pp. 1088-1107, 2014.

[3] D. López, C. Anzola, D. Zapata, E. Rivas, Designing a MAC algorithm for equitable spectrum allocation in cognitive radio wireless networks, Journal Wireless Personal Communications, Volume 98, Issue. 1, pp. 1-32, 2017.

[4] D. López, E. Rivas, O. Gualdron, E, Primary user characterization for cognitive radio wireless networks using a neural system based on Deep Learning, Journal Artificial Intelligence Review (Early Access), pp. 1-27, 2017. doi: 10.1007/s10462-017-9600-4.

[5] D. López, J. Hernández, E. Rivas, SVM and ANFIS as channel selection models for the spectrum decision stage in cognitive radio networks, Journal Contemporary Engineering Science, Volume 10, Issue 10, pp. 475-502, 2017.

[6] J. Guillen, Algoritmo para acceso al medio en redes inalámbricas cognitivas, M.S thesis, Autónoma Metropolitana University, City of Mexico, Mexico, 2012.

[7] J. Chakraborty, V. Varma, M. Erman, ANFIS based opportunistic power control for cognitive radio in spectrum sharing, IEEE Proceeding Electrical Information and Communication Technology (EICT), pp. 1-6, Khulna, Bangladesh, 2014.

[8] G. Ning, X. Cao, X. Duan, K. Chowdhury, K, A spectrum sharing algorithm based on spectrum heterogeneity for centralized cognitive radio networks, Vehicular Technology Conference (VTC Spring), 2011 IEEE 73rd, Hubei, 2011.

[9] M. Benitez, Spectrum usage models for the analysis, design and simulation of cognitive radio networks, Doctoral Thesis, Universidad Politecnica de Catalunya, pp. 6, Spain, 2011.

[10] S. Barnes, Cognitive radio performance optimization through spectrum availability prediction, Thesis Master of Engineering (Electronic Engineering), Department of Electrical, Electronic and Computer Engineering, University of Pretoria, pp. 32, Sudafrica, 2012.

[11] L. Gavrilovska, D. Denkovski, V. Rakovic, M. Angjelichinoski, Medium access control protocol in cognitive radio networks: overview and General Classification, Skopje: IEEE Communication Surveys \& Tutorials, Volume 16, Issue 4, pp. 2092-2124, 2014.

[12] W. Yeol, Spectrum management in cognitive radio wireless networks, Georgia Institute of Technology, Georgia Institute Technology, Georgia, pp. 98-138, 2009.

[13] E. Hernández, Algoritmo para acceso al medio en redes inalámbricas cognitivas, Maestría en Ciencias y Tecnologías de la Información, Universidad Autónoma Metropolitana, México, 2012.

[14] L. Chen, S. Iellamo, M. Coupechoux, P. Godlewski, Spectrum auction with interference constraint for cognitive radio networks with multiple primary and secondary users, Journal Wireless. Networks, Volume. 17, Issue. 5, pp. 1355-1371, 2011.

[15] C. Wang, W. Wang, F. Adachi, Load-balancing spectrum decision for cognitive radio networks, IEEE Journal on Selected Areas in Communications, Volume 10, Issue 2, pp. 161-174, 2011.

[16] J. Chakraborty, V. Varma, M. Erman, ANFIS based opportunistic power control for cognitive radio in spectrum sharing, International Conference on Electrical Information and Communication Technology, Blekinge Institute of Technology, 2013

[17] A. Amraoui, B. Benmammar, F. Krief, F. Bendimerad, Intelligent wireless communication system using cognitive radio, International Journal of Distributed and Parallel Systems, Volume 3, Issue 2, pp. 91-104, 2012.

[18] G. Dioran, Contribution to radio resource and spectrum management strategies in wireless access networks: a Markov modeling approach, Doctoral Dissertation, Universidad Politecnica de Catalunya, Spain, 2010.

[19] C.Anzola, D. Zapata, D, Diseño de un algoritmo MAC para la asignación equitativa de espectro en redes inalámbricas de radio cognitiva, Trabajo de grado, Universidad Distrital Francisco José de Caldas, Bogotá, Colombia, 2016.

[20] G. Büyüközkan, C. Kabraman, D. Ruan, fuzzy multi-criteria decision approach for software development strategy selection, International Journal of General Systems, Vol. 33, No. 2, 259-280, 2004. 